\title{
The drivers and health risks of the unexpected surface ozone enhancements over the Sichuan basin, China in 2020
}

Youwen Sun ${ }^{1,2}$, Hao Yin ${ }^{1,2, \uparrow}$, Xiao Lu ${ }^{3, \dagger}$, Justus Notholt ${ }^{4}$, Mathias Palm${ }^{4}$, Cheng $\mathrm{Liu}^{2}$, Yuan Tian ${ }^{5}$, and Bo Zheng ${ }^{6}$

${ }^{1}$ Key Laboratory of Environmental Optics and Technology, Anhui Institute of Optics and Fine Mechanics, HFIPS, Chinese Academy of Sciences, Hefei 230031, China

${ }^{2}$ Key Laboratory of Precision Scientific Instrumentation of Anhui Higher Education Institutes, University of Science and Technology of China, Hefei, 230026, China

${ }^{3}$ School of Atmospheric Sciences, Sun Yat-sen University, Zhuhai, 519082, China

${ }^{4}$ University of Bremen, Institute of Environmental Physics, P. O. Box 330440, 28334 Bremen, Germany

${ }^{5}$ Institutes of Physical Science and Information Technology, Anhui University, Hefei 230601,

China

${ }^{6}$ Institute of Environment and Ecology, Tsinghua Shenzhen International Graduate School,

Tsinghua University, Shenzhen 518055, China

${ }^{\dagger}$ Corresponding authors.

E-mail addresses: Hao Yin (yhyh95@mail.ustc.edu.cn) and Xiao Lu (luxiao25@mail.sysu.edu.cn) 


\section{Section S1. Metrics definitions}

The root-mean-square error (RMSE), normalized mean bias (NMB), normalized root-meansquare error (NRMSE), and Pearson correlation coefficient (R) are used to evaluate the performance of the GEOS-Chem-XGBoost model. The formulas of these metrics are as follows:

$$
\begin{gathered}
R M S E=\sqrt{\frac{1}{N} \sum_{i=0}^{n}\left(X_{i}-Y_{i}\right)^{2}} \\
N M B=\frac{\sum_{i=0}^{n}\left(X_{i}-Y_{i}\right)}{\sum_{i=0}^{n} Y_{i}} \\
N R M S E=\frac{\sqrt{\frac{1}{N} \sum_{i=0}^{n}\left(X_{i}-Y_{i}\right)^{2}}}{Y_{95 t h}-Y_{5 t h}} \\
R=\frac{N \sum_{i=0}^{n} X_{i} Y_{i}-\sum_{i=0}^{n} X_{i} \cdot \sum_{i=0}^{n} Y_{i}}{\sqrt{N \sum_{i=0}^{n} X_{i}^{2}-\left(\sum_{i=0}^{n} X_{i}\right)^{2}} \cdot \sqrt{N \sum_{i=0}^{n} Y_{i}^{2}-\left(\sum_{i=0}^{n} Y_{i}\right)^{2}}}
\end{gathered}
$$

where $\mathrm{X}$ and $\mathrm{Y}$ represent the datasets of model and measurement, respectively.

Table S1. List of input parameters fed into each XGBoost machine learning model.

\begin{tabular}{ll}
\hline Acronym & Description \\
\hline \multicolumn{1}{c}{ Concentrations of chemical constituents simulated by GEOS-Chem model } \\
\hline $\mathrm{NO}_{2}$ geos & Nitrogen dioxide \\
$\mathrm{NO}$ geos & Nitrogen oxide \\
$\mathrm{NO}_{\text {__geos }}$ & Reactive nitrogen $\left(\mathrm{NO}+\mathrm{NO}_{2}+\right.$ nitrates) \\
$\mathrm{O}_{3}$ geos & Ozone \\
CO_geos & Carbon monoxide \\
ACET_geos & Acetone \\
ALK4_geos & Alkanes \\
ALD2_geos & Acetaldehyde \\
$\mathrm{HCHO}$ _geos & Formaldehyde \\
$\mathrm{C}_{2} \mathrm{H}_{6}$ geos & Ethane \\
$\mathrm{C}_{3} \mathrm{H}_{8}$ geos & Propane \\
$\mathrm{BCP}$ _geos & Hydrophilic black carbon \\
$\mathrm{BCPO}$ _geos & Hydrophobic black carbon \\
$\mathrm{OCPI} g e o s$ & Hydrophilic organic carbon \\
OCPO_geos & Hydrophobic organic carbon \\
EOH_geos & Ethanol \\
DST1_geos & Dust with diameter of $0.7 \mu \mathrm{m}$
\end{tabular}




\begin{tabular}{|c|c|}
\hline DST2_geos & Dust with diameter of $1.4 \mu \mathrm{m}$ \\
\hline DST3_geos & Dust with diameter of $2.4 \mu \mathrm{m}$ \\
\hline DST4_geos & Dust with diameter of $4.5 \mu \mathrm{m}$ \\
\hline $\mathrm{H}_{2} \mathrm{O}_{2}$ _geos & Hydrogen peroxide \\
\hline $\mathrm{HNO}_{3}$ _geos & Nitric acid \\
\hline $\mathrm{HNO}_{4} \_$geos & Pyroxenitic acid \\
\hline ISOP_geos & Isoprene \\
\hline MACR_geos & Methacrolein \\
\hline MEK_geos & Methyl ethyl ketone \\
\hline MVK_geos & Methyl vinyl ketone \\
\hline $\mathrm{N}_{2} \mathrm{O}_{5}$ _geos & Dinitrogen pentoxide \\
\hline $\mathrm{NH}_{3} \_$geos & Ammonia \\
\hline $\mathrm{NH}_{4} \_$geos & Ammonium \\
\hline NIT_geos & Inorganic nitrates \\
\hline PAN_geos & Peroxyacetyl nitrate \\
\hline PRPE_geos & Alkenes \\
\hline $\mathrm{RCHO}$ geos & Aldehyde \\
\hline SALA_geos & Fine sea salt aerosol \\
\hline SALC_geos & Coarse sea salt aerosol \\
\hline $\mathrm{SO}_{2} \_$geos & Sulfur dioxide \\
\hline SOAP_geos & Secondary organic aerosol precursor \\
\hline SOAS_geos & Simple secondary organic aerosol \\
\hline TOLU_geos & Toluene \\
\hline XYLE_geos & Xylene \\
\hline $\mathrm{O}_{\mathrm{x} \_g e o s}$ & Odd oxygen $\left(\mathrm{O}_{3}+\mathrm{NO}_{2}\right)$ \\
\hline $\mathrm{PM}_{2.5 \_ \text {geos }}$ & $\mathrm{PM}_{2.5}$ \\
\hline \multicolumn{2}{|c|}{ Emissions used for GEOS-Chem simulation } \\
\hline ENO & Nitrogen oxide emissions \\
\hline $\mathrm{ECO}$ & Carbon monoxide emissions \\
\hline EACET & Acetone emissions \\
\hline EALD2 & Acetaldehyde emissions \\
\hline EALK4 & Alkanes emissions \\
\hline EBENZ & Benzene emissions \\
\hline $\mathrm{EC}_{2} \mathrm{H}_{6}$ & Ethane emissions \\
\hline EPRPE & Alkenes emissions \\
\hline ETOLU & Toluene emissions \\
\hline EXYLE & Xylene emissions \\
\hline EISOP & Isoprene emissions \\
\hline EBCPI & Hydrophilic black carbon emissions \\
\hline EBCPO & Hydrophobic black carbon emissions \\
\hline EOCPI & Hydrophilic organic carbon emissions \\
\hline EOCPO & Hydrophobic organic carbon emissions \\
\hline ESALA & Fine sea salt aerosol emissions \\
\hline ESALC & Coarse sea salt aerosol emissions \\
\hline
\end{tabular}




\begin{tabular}{ll}
$\mathrm{ESO}_{2}$ & Sulfur dioxide emissions \\
ESOAP & Secondary organic aerosol precursor emissions \\
ESOAS & Simple secondary organic aerosol emissions \\
ECHBR $_{3}$ & Bromoform emissions \\
\hline & Time information \\
\hline Hour & Hour of day \\
Weekday & Day of the week \\
Month & Month of the year \\
Trendday & Days since 1 May 2019 \\
\hline & Meteorological fields used for GEOS-Chem simulation \\
\hline Cldtt & Total cloud fraction \\
Ps & Surface pressure \\
$\mathrm{Q}_{10 \mathrm{~m}}$ & Specific humidity at $10 \mathrm{~m}$ \\
$\mathrm{Q}_{2 \mathrm{~m}}$ & Specific humidity at 2m \\
$\mathrm{T}_{10 \mathrm{~m}}$ & Temperature at 10m \\
$\mathrm{Tprec}$ & Total precipitation \\
$\mathrm{T}$ & Skin surface temperature \\
$\mathrm{T}_{2 \mathrm{~m}}$ & Temperature at 2m \\
$\mathrm{U}_{10 \mathrm{~m}}$ & 10m East-West wind-speed \\
$\mathrm{V}_{10 \mathrm{~m}}$ & 10m North-South wind-speed \\
$\mathrm{U}$ & Skin surface East-West wind-speed \\
$\mathrm{V}$ & Skin surface North-South wind-speed \\
$\mathrm{Zpbl}$ & Planetary boundary layer height \\
\hline &
\end{tabular}

Table S2. Daily $y_{0}$ and $\beta$ values for all non-accidental causes, cardiovascular diseases (CVD), respiratory diseases (RD), hypertension, stroke and chronic obstructive pulmonary disease (COPD). This table is cited from Wang et al. (2021).

\begin{tabular}{lll}
\hline Disease & Daily $y_{0}$ & $\beta(\%)$ \\
\hline All non-accidental causes & $1.687 \times 10^{-5}$ & 0.24 \\
CVD & $3.880 \times 10^{-6}$ & 0.27 \\
RD & $1.841 \times 10^{-6}$ & 0.18 \\
Hypertension & $5.422 \times 10^{-7}$ & 0.60 \\
Stroke & $1.197 \times 10^{-7}$ & 0.29 \\
COPD & $1.623 \times 10^{-6}$ & 0.20 \\
\hline
\end{tabular}

Table S3. Anthropogenic emissions of $\mathrm{NO}_{x}$ and VOCs for Chongqing and Sichuan provinces in May-June 2019 and 2020 provided by the MEE over the SCB (Unit: Tonne)

\begin{tabular}{|l|l|l|l|l|l|l|l|c|}
\hline \multirow{2}{*}{ Pollutant } & \multirow{2}{*}{ Month } & \multirow{2}{*}{ Sector } & \multicolumn{2}{|c|}{2019} & \multicolumn{2}{|c|}{2020} & \multicolumn{2}{c|}{$\%$ Difference } \\
\cline { 3 - 9 } & & & Chongqing & Sichuan & Chongqing & Sichuan & Chongqing & Sichuan \\
\hline & & power & 3971 & 3130 & 6042 & 5048 & $52.15 \%$ & $61.28 \%$ \\
\hline
\end{tabular}




\begin{tabular}{|c|c|c|c|c|c|c|c|c|}
\hline \multirow{11}{*}{$\mathrm{NO}_{x}$} & \multirow{5}{*}{ May } & industry & 11681 & 30494 & 11759 & 29505 & $0.67 \%$ & $-3.24 \%$ \\
\hline & & residential & 956 & 2443 & 970 & 2485 & $1.46 \%$ & $1.72 \%$ \\
\hline & & transportation & 13138 & 21253 & 12290 & 20017 & $-6.45 \%$ & $-5.82 \%$ \\
\hline & & agriculture & 0 & 0 & 0 & 0 & $0 \%$ & $0 \%$ \\
\hline & & total & 29746 & 57320 & 31061 & 57055 & $4.50 \%$ & $-0.46 \%$ \\
\hline & \multirow{6}{*}{ June } & power & 3732 & 3446 & 4814 & 3791 & $28.99 \%$ & $10.01 \%$ \\
\hline & & industry & 13384 & 35479 & 13623 & 34402 & $1.79 \%$ & $-3.04 \%$ \\
\hline & & residential & 924 & 2362 & 937 & 2402 & $1.41 \%$ & $1.69 \%$ \\
\hline & & transportation & 13138 & 21253 & 12300 & 19809 & $-6.38 \%$ & $-6.79 \%$ \\
\hline & & agriculture & 0 & 0 & 0 & 0 & $0 \%$ & $0 \%$ \\
\hline & & total & 31178 & 62540 & 31674 & 60404 & $-1.59 \%$ & $-3.41 \%$ \\
\hline \multirow{12}{*}{ VOCs } & \multirow{6}{*}{ May } & power & 34 & 25 & 52 & 40 & $52.94 \%$ & $60.00 \%$ \\
\hline & & industry & 27938 & 67606 & 27029 & 63614 & $-3.25 \%$ & $-5.90 \%$ \\
\hline & & residential & 6229 & 16419 & 6100 & 16150 & $-2.07 \%$ & $-1.64 \%$ \\
\hline & & transportation & 5732 & 15861 & 5389 & 14348 & $-5.98 \%$ & $-9.54 \%$ \\
\hline & & agriculture & 0 & 0 & 0 & 0 & $0 \%$ & $0 \%$ \\
\hline & & total & 39933 & 99911 & 38570 & 94152 & $-3.41 \%$ & $-5.67 \%$ \\
\hline & \multirow{6}{*}{ June } & power & 32 & 27 & 41 & 30 & $28.13 \%$ & $11.11 \%$ \\
\hline & & industry & 34528 & 83393 & 33523 & 78503 & $-2.91 \%$ & $-5.86 \%$ \\
\hline & & residential & 6069 & 15967 & 5946 & 15708 & $-2.03 \%$ & $-1.62 \%$ \\
\hline & & transportation & 5732 & 15861 & 6016 & 16211 & $4.95 \%$ & $2.21 \%$ \\
\hline & & agriculture & 0 & 0 & 0 & 0 & $0 \%$ & $0 \%$ \\
\hline & & total & 46361 & 115248 & 45526 & 110452 & $-1.80 \%$ & $-4.16 \%$ \\
\hline
\end{tabular}

Table S4. Total daily mortalities from all non-accidental causes (NAC), CVD, RD, COPD, hypertension (HT), and stroke attributable to ambient $\mathrm{O}_{3}$ exposure in each city over the $\mathrm{SCB}$ within May-June and the whole year in 2019.

\begin{tabular}{l|l|l|l|l|l|l|l}
\hline City & Period & NAC & CVD & RD & HT & Stroke & COPD \\
\hline \multirow{3}{*}{ Abazhou } & Jan-Dec & 86 & 22 & 7 & 7 & 1 & 7 \\
\cline { 2 - 8 } Bazhong & May-Jun & 8 & 2 & 1 & 1 & 0 & 1 \\
\hline \multirow{3}{*}{ Chengdu } & Jan-Dec & 271 & 70 & 22 & 21 & 2 & 22 \\
\cline { 2 - 8 } & May-Jun & 49 & 13 & 4 & 4 & 0 & 4 \\
\hline \multirow{3}{*}{ Chongqing } & Jan-Dec & 3427 & 882 & 283 & 259 & 29 & 277 \\
\cline { 2 - 8 } & May-Jun & 850 & 219 & 70 & 66 & 7 & 68 \\
\hline \multirow{3}{*}{ Dazhou } & Jan-Dec & 4851 & 1248 & 402 & 365 & 41 & 392 \\
\cline { 2 - 8 } & May-Jun & 415 & 107 & 34 & 32 & 4 & 33 \\
\hline \multirow{3}{*}{ Deyang } & Jan-Dec & 564 & 145 & 46 & 43 & 5 & 45 \\
\cline { 2 - 8 } & May-Jun & 31 & 8 & 3 & 2 & 0 & 2 \\
\hline \multirow{3}{*}{ Ganzizhou } & Jan-Dec & 529 & 136 & 44 & 40 & 4 & 43 \\
\cline { 2 - 8 } & May-Jun & 124 & 32 & 10 & 10 & 1 & 10 \\
\hline \multirow{2}{*}{ Guangan } & Jan-Dec & 31 & 8 & 3 & 2 & 0 & 2 \\
\hline & May-Jun & 0 & 0 & 0 & 0 & 0 & 0 \\
\hline
\end{tabular}




\begin{tabular}{|c|c|c|c|c|c|c|c|}
\hline & May-Jun & 80 & 21 & 7 & 6 & 1 & 6 \\
\hline \multirow{2}{*}{ Guangyuan } & Jan-Dec & 141 & 36 & 12 & 11 & 1 & 11 \\
\hline & May-Jun & 10 & 3 & 1 & 1 & 0 & 1 \\
\hline \multirow{2}{*}{ Leshan } & Jan-Dec & 442 & 114 & 36 & 34 & 4 & 36 \\
\hline & May-Jun & 87 & 22 & 7 & 7 & 1 & 7 \\
\hline \multirow{2}{*}{ Liangshanzhou } & Jan-Dec & 934 & 241 & 77 & 71 & 8 & 75 \\
\hline & May-Jun & 246 & 63 & 20 & 19 & 2 & 20 \\
\hline \multirow{2}{*}{ Luzhou } & Jan-Dec & 559 & 144 & 46 & 42 & 5 & 45 \\
\hline & May-Jun & 40 & 10 & 3 & 3 & 0 & 3 \\
\hline \multirow{2}{*}{ Meishan } & Jan-Dec & 571 & 147 & 47 & 43 & 5 & 46 \\
\hline & May-Jun & 142 & 36 & 12 & 11 & 1 & 11 \\
\hline \multirow{2}{*}{ Mianyang } & Jan-Dec & 664 & 171 & 55 & 51 & 6 & 54 \\
\hline & May-Jun & 164 & 42 & 13 & 13 & 1 & 13 \\
\hline \multirow{2}{*}{ Nanchong } & Jan-Dec & 656 & 169 & 54 & 50 & 6 & 53 \\
\hline & May-Jun & 134 & 35 & 11 & 11 & 1 & 11 \\
\hline \multirow{2}{*}{ Neijiang } & Jan-Dec & 407 & 105 & 34 & 31 & 3 & 33 \\
\hline & May-Jun & 60 & 15 & 5 & 5 & 1 & 5 \\
\hline \multirow{2}{*}{ Panzhihua } & Jan-Dec & 208 & 54 & 17 & 16 & 2 & 17 \\
\hline & May-Jun & 62 & 16 & 5 & 5 & 1 & 5 \\
\hline \multirow{2}{*}{ Suining } & Jan-Dec & 353 & 91 & 29 & 27 & 3 & 28 \\
\hline & May-Jun & 60 & 16 & 5 & 5 & 1 & 5 \\
\hline \multirow{2}{*}{ Yaan } & Jan-Dec & 170 & 44 & 14 & 13 & 1 & 14 \\
\hline & May-Jun & 33 & 8 & 3 & 3 & 0 & 3 \\
\hline \multirow{2}{*}{ Yibin } & Jan-Dec & 734 & 189 & 61 & 56 & 6 & 59 \\
\hline & May-Jun & 123 & 32 & 10 & 10 & 1 & 10 \\
\hline \multirow{2}{*}{ Zigong } & Jan-Dec & 391 & 101 & 32 & 30 & 3 & 32 \\
\hline & May-Jun & 71 & 18 & 6 & 6 & 1 & 6 \\
\hline \multirow{2}{*}{ Ziyang } & Jan-Dec & 371 & 96 & 31 & 28 & 3 & 30 \\
\hline & May-Jun & 87 & 22 & 7 & 7 & 1 & 7 \\
\hline \multirow{2}{*}{ Total } & Jan-Dec & 16773 & 4319 & 1386 & 1272 & 143 & 1354 \\
\hline & May-Jun & 2874 & 741 & 237 & 223 & 25 & 231 \\
\hline
\end{tabular}

Table S5. Total daily mortalities from all non-accidental causes (NAC), CVD, RD, COPD, hypertension (HT), and stroke attributable to ambient $\mathrm{O}_{3}$ exposure in each city over the SCB within May-June and the whole year in 2020.

\begin{tabular}{l|l|l|l|l|l|l|l}
\hline City & Peoriod & NAC & CVD & RD & HT & Stroke & COPD \\
\hline \multirow{3}{*}{ Abazhou } & Jan-Dec & 75 & 19 & 6 & 6 & 1 & 6 \\
\cline { 2 - 8 } & May-Jun & 11 & 3 & 1 & 1 & 0 & 1 \\
\hline \multirow{3}{*}{ Bazhong } & Jan-Dec & 273 & 70 & 22 & 21 & 2 & 22 \\
\cline { 2 - 8 } & May-Jun & 95 & 25 & 8 & 7 & 1 & 8 \\
\hline \multirow{2}{*}{ Chengdu } & Jan-Dec & 4376 & 1126 & 362 & 328 & 37 & 354 \\
\cline { 2 - 8 } & May-Jun & 1488 & 383 & 123 & 113 & 13 & 120 \\
\hline Chongqing & Jan-Dec & 4374 & 1126 & 362 & 331 & 37 & 353 \\
\hline
\end{tabular}




\begin{tabular}{|c|c|c|c|c|c|c|c|}
\hline & May-Jun & 1070 & 276 & 88 & 83 & 9 & 86 \\
\hline \multirow{2}{*}{ Dazhou } & Jan-Dec & 411 & 106 & 34 & 32 & 4 & 33 \\
\hline & May-Jun & 57 & 15 & 5 & 4 & 0 & 5 \\
\hline \multirow{2}{*}{ Deyang } & Jan-Dec & 657 & 169 & 54 & 50 & 6 & 53 \\
\hline & May-Jun & 220 & 57 & 18 & 17 & 2 & 18 \\
\hline \multirow{2}{*}{ Ganzizhou } & Jan-Dec & 59 & 15 & 5 & 5 & 1 & 5 \\
\hline & May-Jun & 10 & 2 & 1 & 1 & 0 & 1 \\
\hline \multirow{2}{*}{ Guangan } & Jan-Dec & 522 & 135 & 43 & 40 & 4 & 42 \\
\hline & May-Jun & 156 & 40 & 13 & 12 & 1 & 13 \\
\hline \multirow{2}{*}{ Guangyuan } & Jan-Dec & 287 & 74 & 24 & 22 & 2 & 23 \\
\hline & May-Jun & 110 & 28 & 9 & 8 & 1 & 9 \\
\hline \multirow{2}{*}{ Leshan } & Jan-Dec & 546 & 141 & 45 & 41 & 5 & 44 \\
\hline & May-Jun & 181 & 47 & 15 & 14 & 2 & 15 \\
\hline \multirow{2}{*}{ Liangshanzhou } & Jan-Dec & 814 & 210 & 67 & 63 & 7 & 66 \\
\hline & May-Jun & 183 & 47 & 15 & 14 & 2 & 15 \\
\hline \multirow{2}{*}{ Luzhou } & Jan-Dec & 674 & 174 & 56 & 51 & 6 & 54 \\
\hline & May-Jun & 164 & 42 & 13 & 13 & 1 & 13 \\
\hline \multirow{2}{*}{ Meishan } & Jan-Dec & 639 & 164 & 53 & 48 & 5 & 52 \\
\hline & May-Jun & 205 & 53 & 17 & 16 & 2 & 17 \\
\hline \multirow{2}{*}{ Mianyang } & Jan-Dec & 868 & 224 & 72 & 66 & 7 & 70 \\
\hline & May-Jun & 304 & 78 & 25 & 23 & 3 & 24 \\
\hline \multirow{2}{*}{ Nanchong } & Jan-Dec & 595 & 154 & 49 & 46 & 5 & 48 \\
\hline & May-Jun & 174 & 45 & 14 & 14 & 1 & 14 \\
\hline \multirow{2}{*}{ Neijiang } & Jan-Dec & 547 & 141 & 45 & 42 & 5 & 44 \\
\hline & May-Jun & 172 & 44 & 14 & 13 & 1 & 14 \\
\hline \multirow{2}{*}{ Panzhihua } & Jan-Dec & 178 & 46 & 15 & 14 & 2 & 14 \\
\hline & May-Jun & 47 & 12 & 4 & 4 & 0 & 4 \\
\hline \multirow{2}{*}{ Suining } & Jan-Dec & 422 & 109 & 35 & 32 & 4 & 34 \\
\hline & May-Jun & 132 & 34 & 11 & 10 & 1 & 11 \\
\hline \multirow{2}{*}{ Yaan } & Jan-Dec & 174 & 45 & 14 & 13 & 1 & 14 \\
\hline & May-Jun & 63 & 16 & 5 & 5 & 1 & 5 \\
\hline \multirow{2}{*}{ Yibin } & Jan-Dec & 839 & 216 & 69 & 64 & 7 & 68 \\
\hline & May-Jun & 269 & 69 & 22 & 20 & 2 & 22 \\
\hline \multirow{2}{*}{ Zigong } & Jan-Dec & 503 & 129 & 42 & 38 & 4 & 41 \\
\hline & May-Jun & 169 & 44 & 14 & 13 & 1 & 14 \\
\hline \multirow{2}{*}{ Ziyang } & Jan-Dec & 465 & 120 & 38 & 35 & 4 & 38 \\
\hline & May-Jun & 177 & 46 & 15 & 13 & 2 & 14 \\
\hline \multirow{2}{*}{ Total } & Jan-Dec & 18301 & 4712 & 1513 & 1387 & 156 & 1477 \\
\hline & May-Jun & 5455 & 1406 & 450 & 418 & 46 & 440 \\
\hline
\end{tabular}




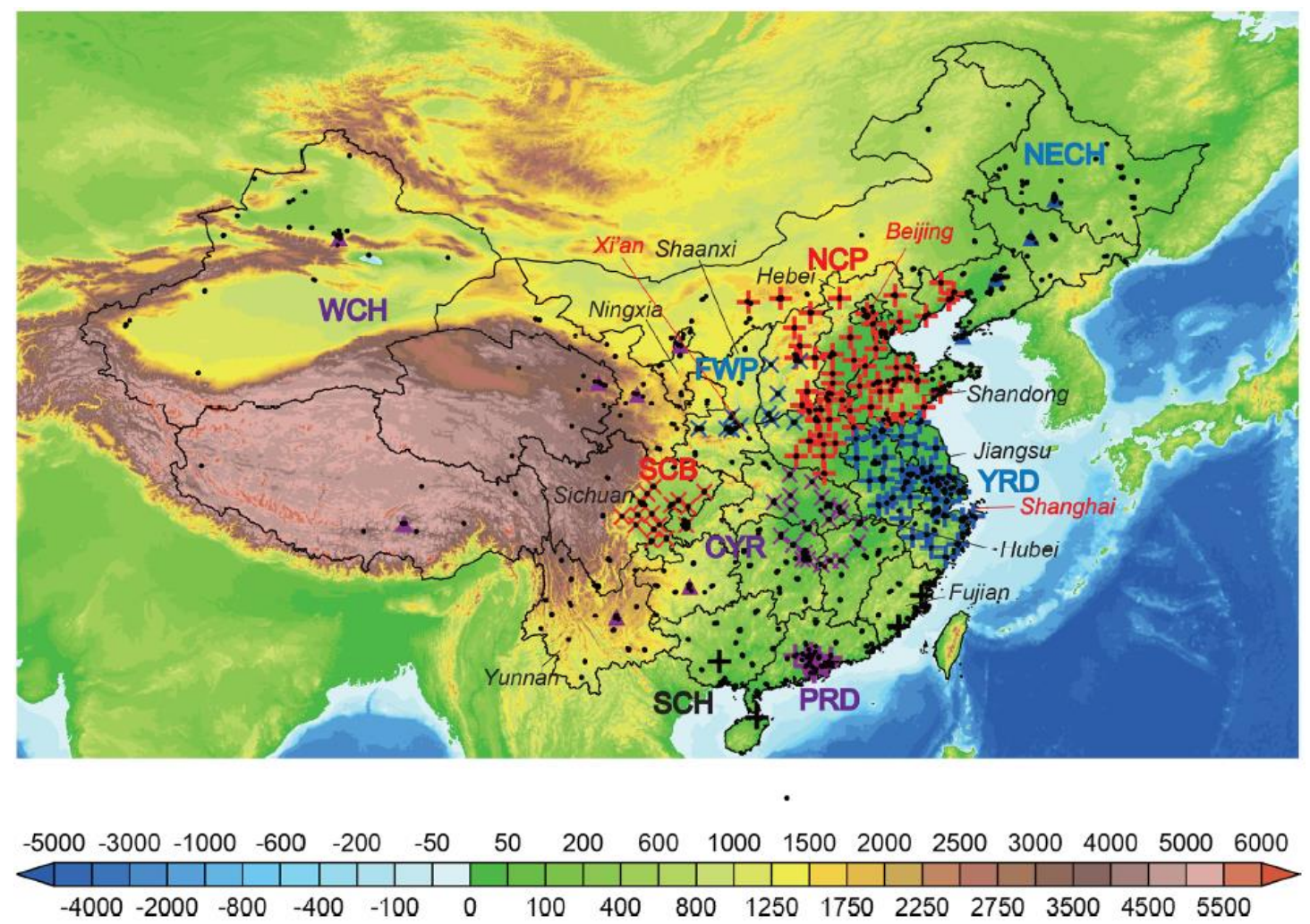

Figure S1. Site locations (black dots) of the China National Environmental Monitoring Center (CNEMC) network. Also shown are the locations of 169 major cities grouped to 9 city clusters: the North China Plain (NCP; red pluses) cluster, the Yangtze River Delta (YRD; blue pluses) cluster, the Fenwei Plain (FWP; blue crosses) cluster, the Sichuan Basin (SCB; red crosses) cluster, the central Yangtze River (CYR; purple crosses) cluster, the Pearl River Delta (PRD; purple pluses) cluster, the northeastern China (NECH; blue triangles) cluster, the western China (WCH; purple triangles) cluster, and southern China ( $\mathrm{SCH}$; black pluses). The underlying figure shows terrain elevation $(\mathrm{m})$. This figure is cited from Lu et al. (2019).

(a) VOC Emissions

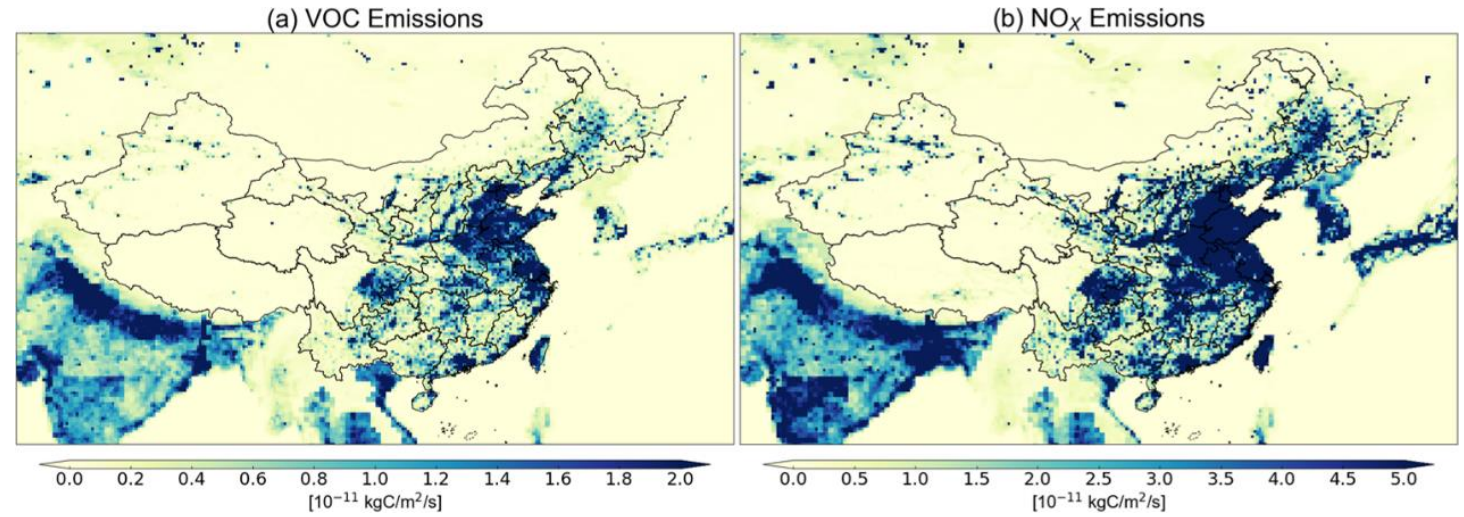

Figure S2. The distribution of anthropogenic emissions of VOCs and $\mathrm{NO}_{\mathrm{x}}$ across China prescribed from the MEIC inventory v2017. 


$$
\Gamma
$$


(a) MDA8 $\mathrm{O}_{3}$ vs $\mathrm{T}_{\text {MAX }}$

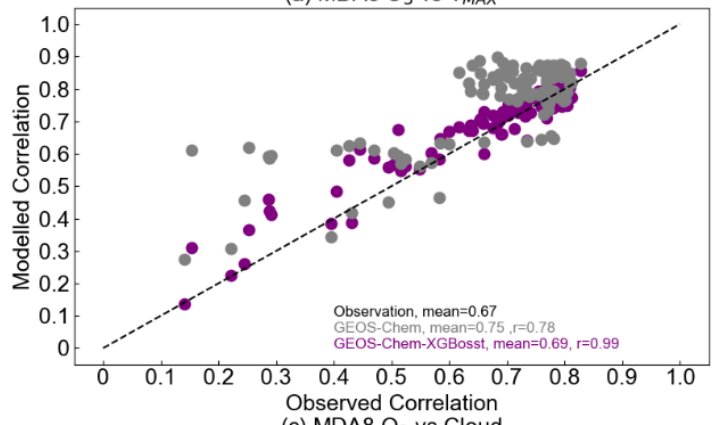
(c) MDA8 O3 vs Cloud

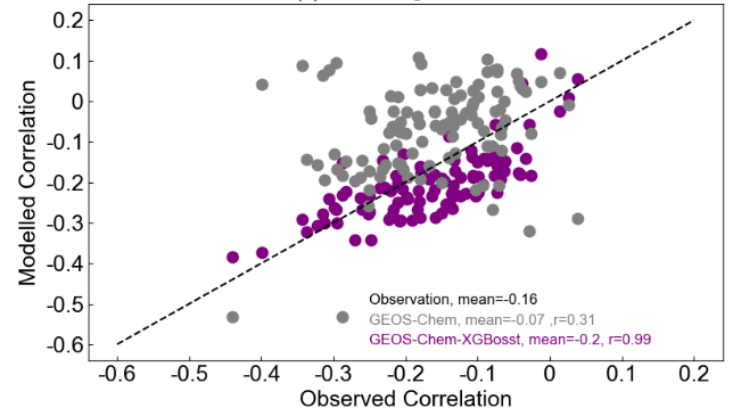

(b) $\mathrm{MDA} 8 \mathrm{O}_{3}$ vs Specific humidity
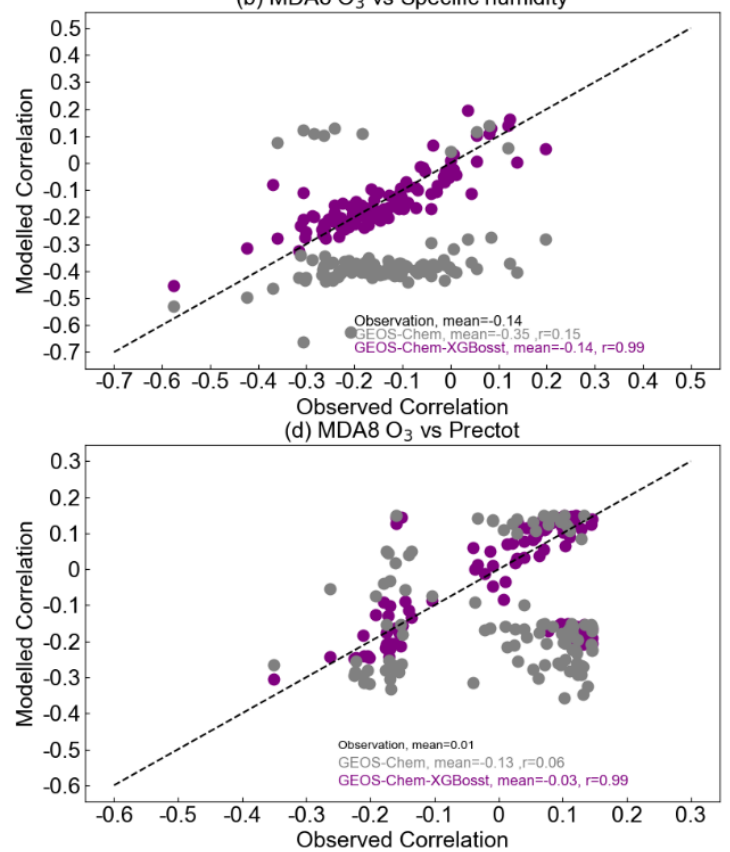

Figure S5. Measured and modelled correlation coefficients between deseasonalized surface MDA8 ozone and daily maximum $2 \mathrm{~m}$ temperature $r_{\mathrm{ozone}, \mathrm{T}}(\mathrm{a})$, specific humidity $r_{\mathrm{ozone}, \mathrm{H}}(\mathrm{b})$, cloud fraction $r_{\mathrm{ozone}, \mathrm{C}}$ (c), and precipitation $r_{\mathrm{ozone}, \mathrm{P}}$

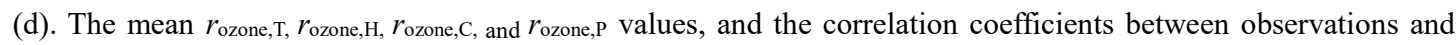
GEOS-Chem and GEOS-Chem-XGBoost results are shown inset.

(a) Chengdu
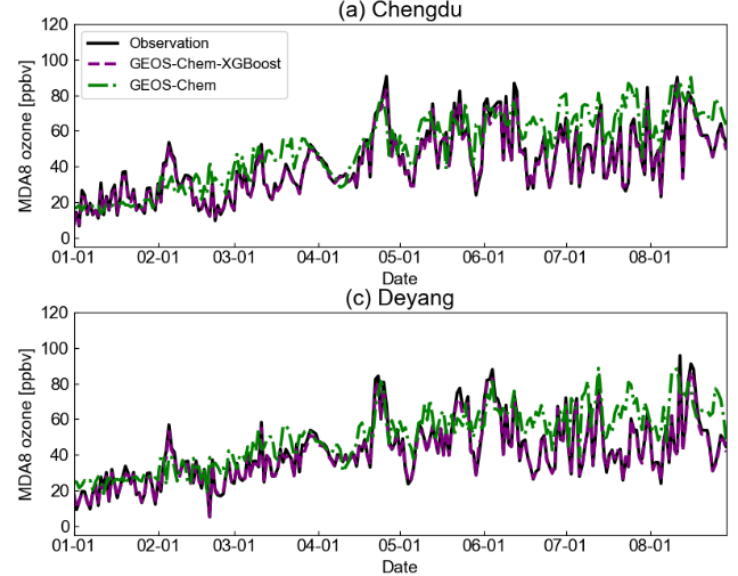

(e) Nanchong

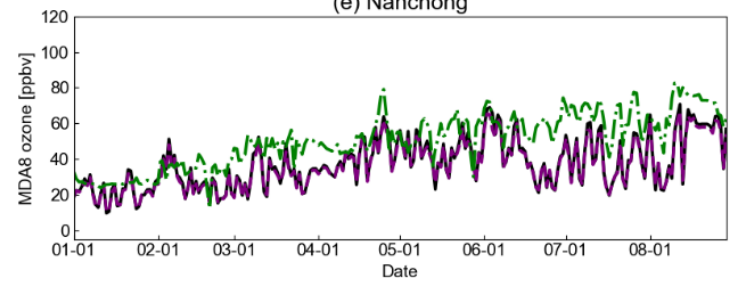

(b) Chongqing
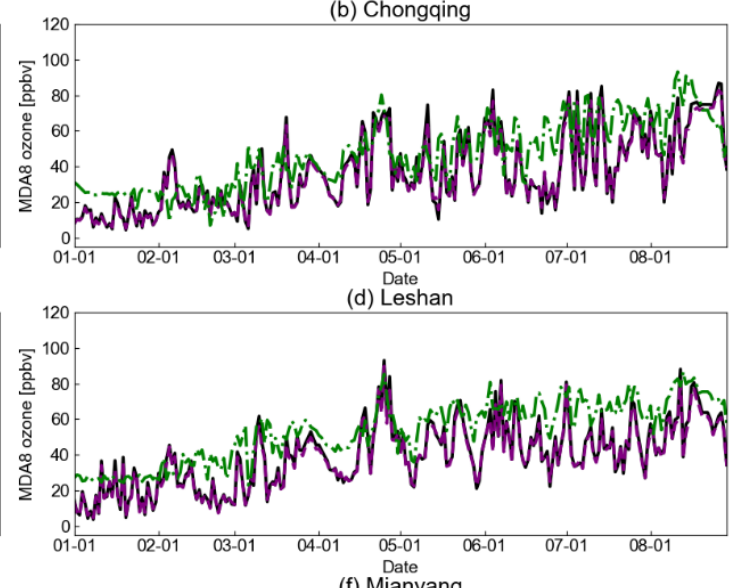
(f) Mianyang

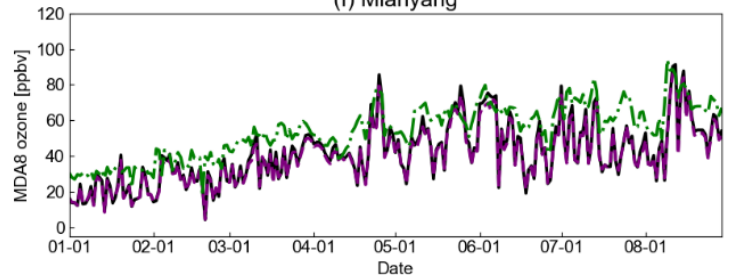

Figure S6. Measured and modelled $\mathrm{O}_{3}$ variabilities in the selected cities over the SCB in 2019. Measured, GEOSChem, and GEOS-Chem-XGBoost predicted $\mathrm{O}_{3}$ concentrations are denoted by black solid, green dashed, and purple dashed lines, respectively. (a) Chengdu, (b) Chongqing, (c) Deyang, (d) Leshan, (e) Nanchong, and (e) Mianyang. 
(a) $2019 \mathrm{HCHO} / \mathrm{NO}_{2}$

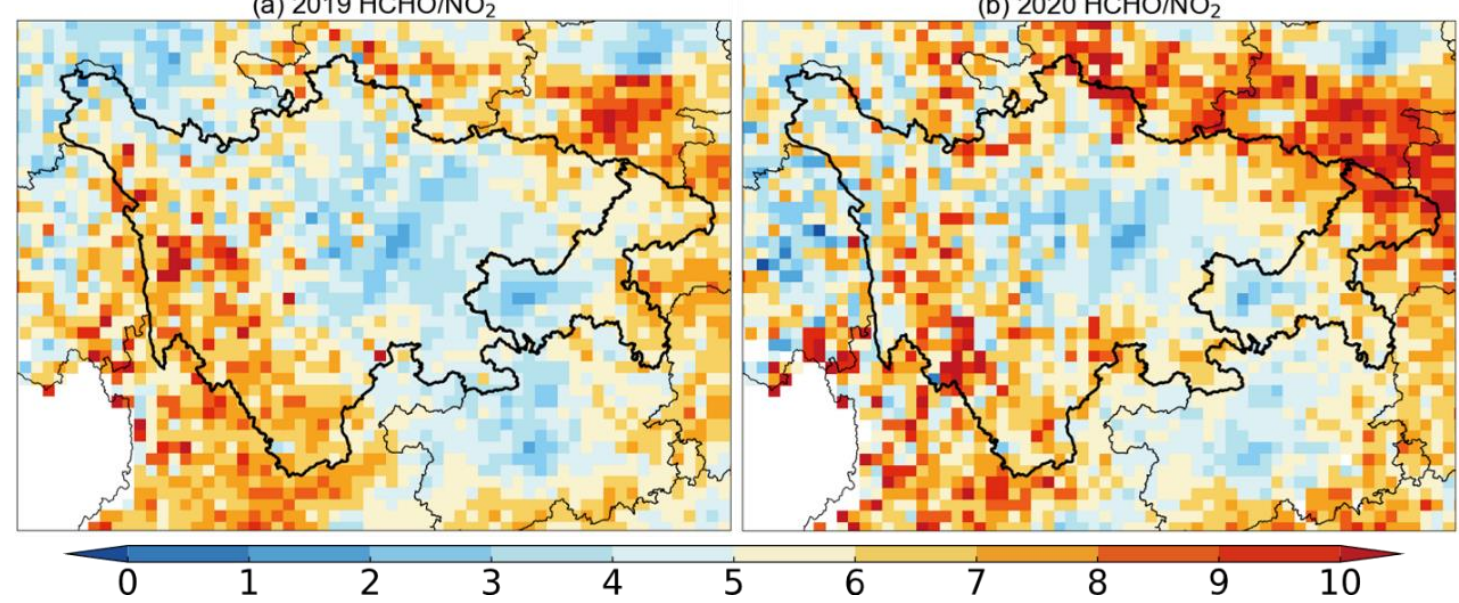

Figure S7. May-June mean TROPOMI HCHO/NO $\mathrm{Na}_{2}$ ratios between (a) 2019 and (b) 2020. The $\mathrm{HCHO} / \mathrm{NO}_{2}$ ratios in May-June in most cities over the SCB have indicated a shift toward high values from 2019 to 2020 but the $\mathrm{O}_{3}$ chemical sensitivities in 2020 in most cities still lie within the transitional regime between 1 and 7 .

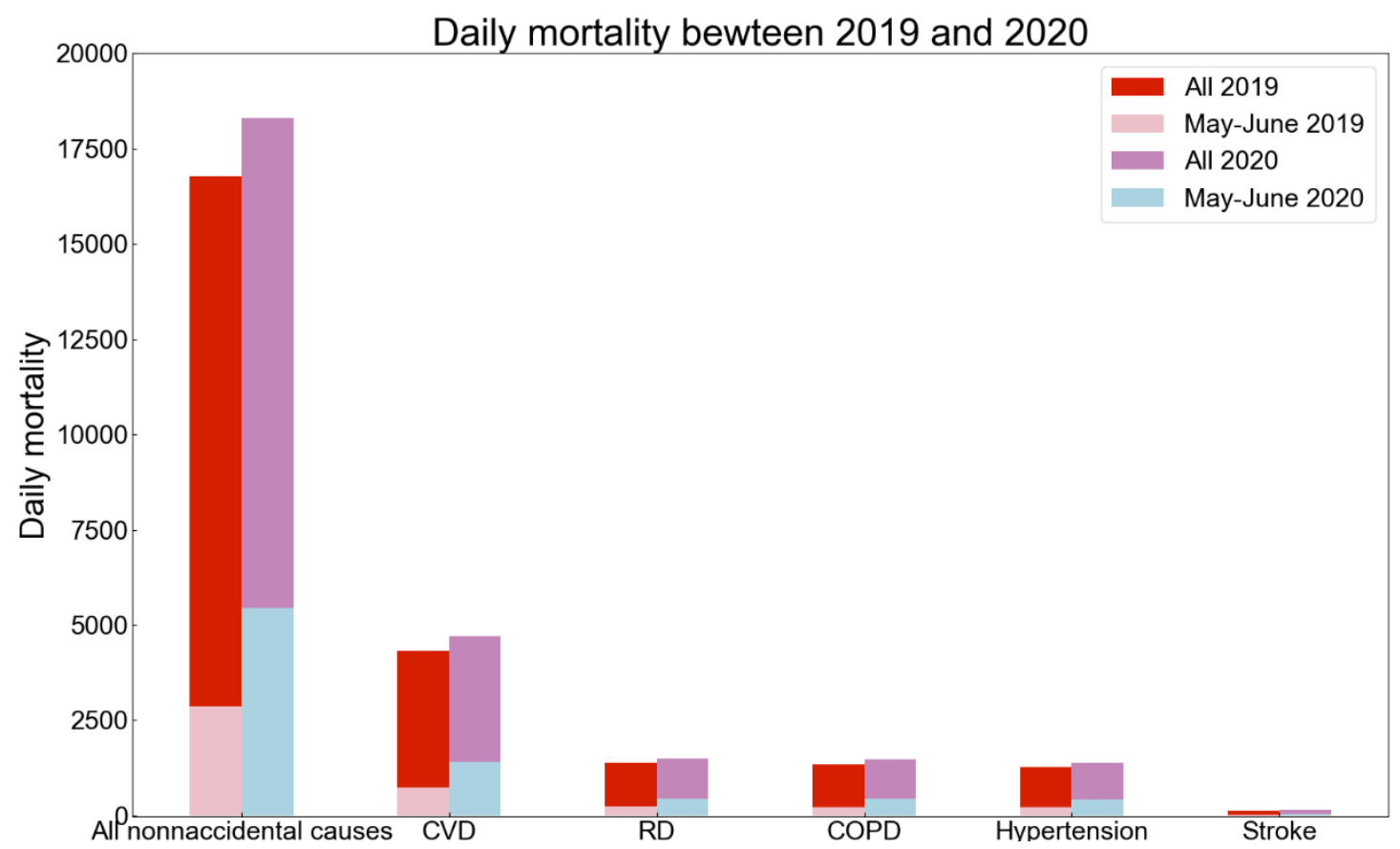

Figure S8. Total daily mortality from all non-accidental causes, CVD, RD, COPD, hypertension, and stroke attributable to ambient $\mathrm{O}_{3}$ exposure in all cities over the SCB within May-June and the whole year in 2019 and 2020. 


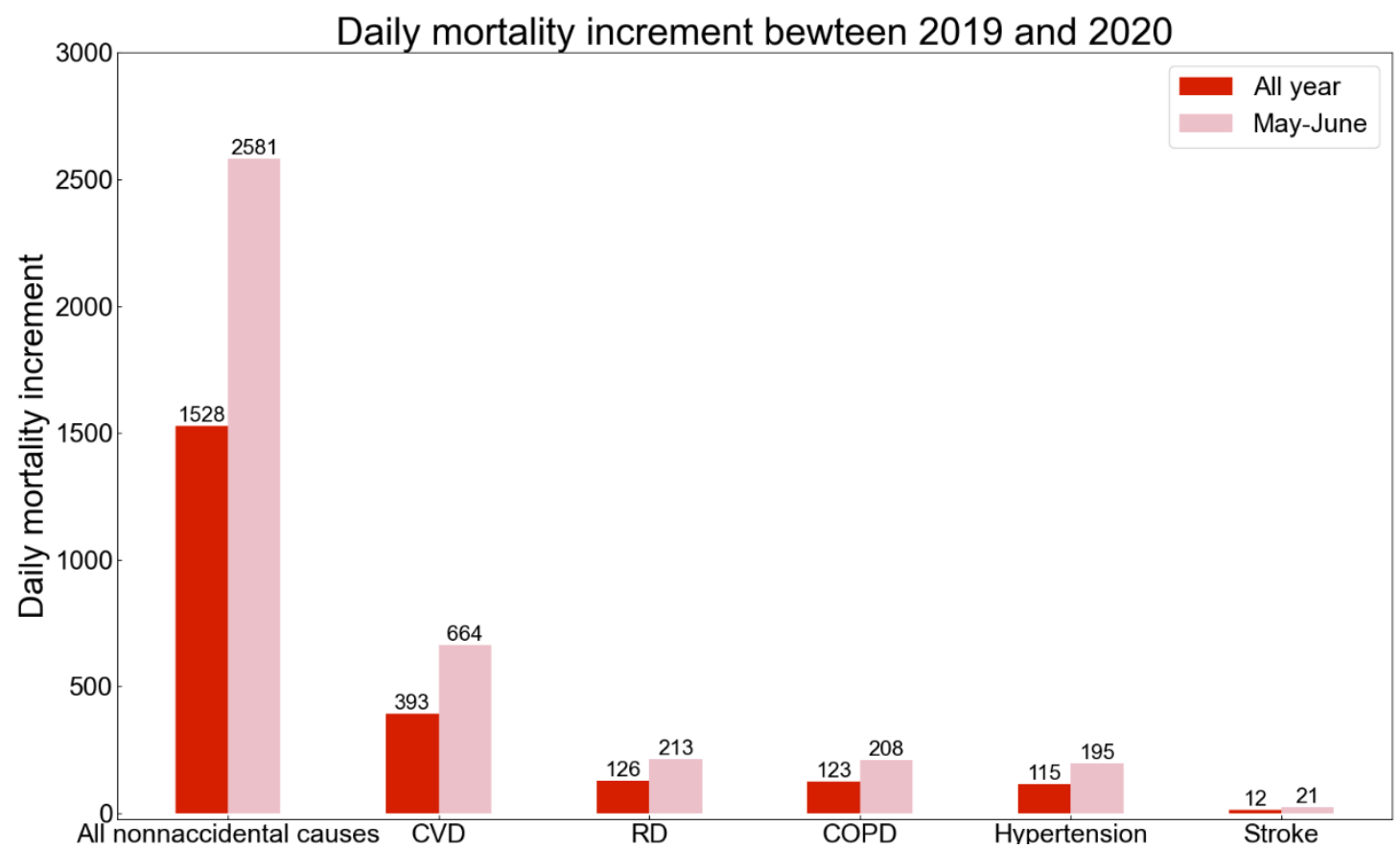

Figure S9. Difference in total daily mortality from all non-accidental causes, CVD, RD, COPD, hypertension, and stroke attributable to ambient $\mathrm{O}_{3}$ exposure in all cities over the SCB within May-June and the whole year between 2019 and 2020 